\title{
Emotionsregulation verbessert die Erfolgschancen
}

Fragestellung: Welchen Stellenwert haben emotionsregulatorische Fertigkeiten für den Erfolg der Psychotherapie?

Hintergrund: Die Erforschung emotionsregulatorischer Techniken in der Psychotherapie hat in den letzten Jahren rapide zugenommen. Empirische Studien zeigen die Bedeutung der Aktivierung und Bearbeitung von emotionalen Konflikten beziehungsweise die Förderung emotionsregulatorischer Fertigkeiten als einen wesentlichen Wirkfaktor der Psychotherapie.

Patienten und Methodik: Bei 118 Patienten mit einer affektiven Störung, einer Angststörung oder einer Somatisierungsstörung wurden bei der stationären Aufnahme und vor der Entlassung spezifische Fertigkeiten mit dem Interview zur „Operationalisierten Fertigkeitsdiagnostik" (OFD) untersucht. $\mathrm{Zu}$ den untersuchten Fertigkeiten gehörten Emotionsregulation, Problemlösung, soziale Kom- petenz, Stressmanagement, Entspannungsverfahren, Selbstwirksamkeit und Arbeit am Selbstwertgefühl. Das primäre Erfolgsmaß war die Ausprägung der depressiven Symptomatik, gemessen mit dem „Beck Depressions Inventar-II“(BDI-II). Die Ergebnisse des OFD und des BDI wurden mittels einer Regressionsanalyse verglichen.

Ergebnisse: In dieser Psychotherapiestudie wurde eine Assoziation von emotionsregulatorischen Fertigkeiten vor der Behandlung mit der Verbesserung der depressiven Beschwerden unter einer stationären Psychotherapie bei Patienten mit affektiven Störungen gefunden. Diese emotionsregulatorischen Fertigkeiten waren auch eine Moderatorvariable für die Assoziation der Verbesserung der anderen Fertigkeiten, gemessen mit dem OFD, im Laufe der Therapie mit der Reduktion depressiver Symptome am Ende der Therapie.

Schlussfolgerungen: Emotionsregulatorische Fertigkeiten sind ein zentraler Wirkfaktor beziehungsweise Mediator in der Psychotherapie depressiver Beschwerden und sollten in psychotherapeutischen Verfahren eine verstärkte Beachtung bekommen.

\section{-Kommentar von Claas-Hinrich Lammers, Hamburg}

\section{Bedeutung emotionsregulierender Faktoren bestätigt}

Die Frage nach den Wirkfaktoren in der Psychotherapie ist von eminenter Bedeutung für das Verständnis psychotherapeutischer Prozesse und die Neukonzeption von Psychotherapien. Diese Studie weist nach, dass emotionsregulatorische Fertigkeiten der Patienten vor Beginn der Psychotherapie signifikant mit der Besserung der depressiven Symptomatik von Patienten im Verlauf der stationären Psychotherapie assoziiert sind. Andere Fertigkeiten vor Beginn der Therapie wie Problemlösung, soziale Kompetenz, Stressmanagement, Entspannungsverfahren, Selbstwirksamkeit und Arbeit am Selbstwertgefühl hatten demgegenüber keinen oder nur geringen Einfluss auf das Ergebnis der Psychotherapie. Demnach sind vom OFD erfasste emotionsregulatorische Techniken wie "Identifikation von Emotionen", ,Akzeptanz von Emotionen", "Regulation von Emotionen" und "Ausdruck von Emotionen" von besonderer Bedeutung für die psychotherapeutische Arbeit an depressiven Beschwerden. Der Studie zufolge haben emotionsregulatorische Fertigkeiten auch eine wichtige Funktion als Moderatorvariable für den positiven Einfluss der anderen genannten Fertigkeiten auf die depressiven Beschwerden, so dass ein Training der Emotionsregulation unerlässlich erscheint. Allein aus diesem Grund hat diese Studie eine hohe praktische Relevanz und deckt sich mit den Ergebnissen einer Vielzahl anderer Stu- dien, welche die Bedeutung emotionsbezogener Techniken und Interventionen in der Psychotherapie betonen. Das Studiendesign ist jedoch auch kritisch zu betrachten, da eine diagnostisch heterogene Patientengruppe untersucht wurde (affektive Störungen, Angststörungen, Somatisierungsstörungen). Bezüglich der Standardisierung der Behandlungsmethoden ist auch Kritik angebracht, da in den verschiedenen Studienzentren unterschiedliche Therapien angewendet wurden, deren Zusammenhang mit den Ergebnissen bezüglich der Emotionsregulation unklar bleiben muss.

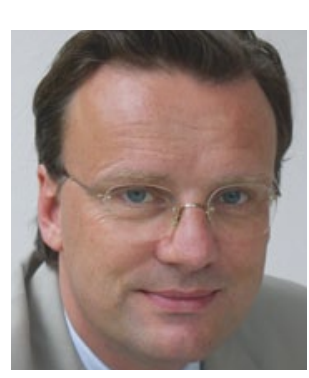

Prof. Dr. med. Claas-Hinrich Lammers, Hamburg

Ärztlicher Direktor und Chefarzt der I. und III. Klinik

Asklepios Klinik Nord - Ochsenzoll, Hamburg

E-Mail: c.lammers@asklepios.com 